\title{
USO DEL ÍNDICE DE ÁREA FOLIAR Y DEL PORCENTAJE DE COBERTURA DEL SUELO PARA ESTIMAR LA RADIACIÓN INTERCEPTADA EN PAPA
}

\section{Use of leaf area index and ground cover to estimate intercepted radiation in potato}

\author{
Antonio de la Casa ${ }^{1}$ *, Gustavo Ovando ${ }^{1}$, Luciano Bressanini ${ }^{1}$, \\ Ángel Rodríguez ${ }^{1}$ y Jorge Martínez ${ }^{1}$.
}

\begin{abstract}
A B S T R A C T
Since there is no information in the green belt of Córdoba, Argentine, about the use of instruments to measure leaf area index (IAF) through light interception in potato crop (Solanum tuberosum L.), a study was developed with the objectives of: i) evaluating the employment and capacity of an optic instrument to determine IAF in a potato crop; ii) verifying IAF and ground cover $(\% \mathrm{C})$ using alternative measurements of crop canopy development to estimate intercepted radiation (fAPAR). The measurements were carried out during 2005 (from February 4 to June 1 ), in a plantation density experiment near Córdoba ( $31^{\circ} 30^{\prime} \mathrm{S}$ lat, $64^{\circ} 08^{\prime} \mathrm{W}$ long, 402 m.a.s.l.), with three treatments, in $\mathrm{pl} \mathrm{m}^{-2}: 6.0 \pm 0.6$ (TDC); $3.9 \pm 0.2$ (TDB) and $7.9 \pm 0.7$ (TDA). IAF was estimated by means of an optic instrument (ceptometer) and ground cover $(\% \mathrm{C})$ through digital pictures. The IAF values measured with the ceptometer were compared with photogrametric determinations obtaining an acceptable correlation $\left(\mathrm{R}^{2}=0.80 ; \mathrm{P}<0.01\right)$. The relationship between IAF and fAPAR remained uniform, in the sense of comparing plantation densities, likewise during different moments of the cycle. Until the crop reaches maximum covering, the relationship between $\% \mathrm{C}$ and fAPAR was linear, with a highly significant $\left(\mathrm{R}^{2}=0.93 ; \mathrm{P}<0.01\right)$ coefficient of adjustment, although it shifts slightly away from a 1:1 relationship.
\end{abstract}

Key words: potato, plant density, ground cover, intercepted radiation, Solanum tuberosum L.

\section{R E S U M E N}

Debido a que no existen antecedentes en el cinturón verde de Córdoba, Argentina, del uso de aparatos para medir el índice de área foliar (IAF) a través de la intercepción lumínica en el cultivo de papa (Solanum tuberosum L.), se desarrolló un trabajo con estos objetivos: i) evaluar el empleo y capacidad de un instrumento óptico para determinar el IAF; ii) verificar la utilización del IAF y de la cobertura del suelo como mediciones alternativas del desarrollo de la parte aérea del cultivo, a fin de estimar la fracción de radiación interceptada (fAPAR). Las mediciones se realizaron durante la campaña de papa semitardía del año 2005 (del 4 de febrero al 1 de junio), en un ensayo de densidad de plantación ubicado próximo a la ciudad de Córdoba ( $31^{\circ} 30^{\prime}$ lat. Sur, $64^{\circ} 08^{\prime}$ long. Oeste, 402 m.s.n.m.), que consistió de tres tratamientos, en $\mathrm{pl} \mathrm{m}^{-2}$ : $6,0 \pm 0,6(\mathrm{TDC}) ; 3,9 \pm 0,2(\mathrm{TDB})$ y $7,9 \pm 0,7(\mathrm{TDA})$. El IAF se midió por medio de un interceptómetro óptico y el porcentaje de cobertura del suelo $(\% \mathrm{C})$ a partir de fotografías digitales. Los valores de IAF calculados con las mediciones del interceptómetro se contrastaron con determinaciones fotogramétricas, obteniendo una correlación aceptable $\left(\mathrm{R}^{2}=0,80 ; \mathrm{P}<\right.$ $0,01)$ entre ambos. La relación entre IAF y fAPAR se mantuvo uniforme, tanto en el sentido de comparar las densidades de plantación, como así también durante diferentes momentos del ciclo. Hasta que el cultivo alcanza cobertura máxima, la relación entre $\% \mathrm{C}$ y fAPAR es lineal, con un coeficiente de ajuste altamente significativo $\left(R^{2}=0,93 ; P<0,01\right)$, si bien se aparta ligeramente de la relación 1:1.

Palabras clave: papa, densidad de plantación, IAF, cobertura, radiación interceptada, Solanum tuberosum L.

\footnotetext{
${ }^{1}$ Universidad Nacional de Córdoba, Facultad de Ciencias Agropecuarias, CC 509-Ciudad Universitaria, 5000 Córdoba, Argentina. E-mail: delacasa@agro.uncor.edu *Autor para correspondencia.

Recibido: 26 de diciembre de 2005. Aceptado: 22 de mayo de 2006.
} 


\section{INTRODUCCIÓN}

El cultivo de papa (Solanum tuberosum L.) constituye uno de los principales rubros de la producción hortícola en el cinturón verde de la ciudad de Córdoba (Lanfranconi et al., 1987), donde resulta habitual encontrar lotes (potreros) con diferente grado de heterogeneidad en la cobertura del suelo durante el ciclo de producción, como así también en el rendimiento de tubérculo a la cosecha.

La mayoría de los modelos de rendimiento de un cultivo, siguiendo la propuesta de Monteith (1977, 1994), estiman la producción máxima de biomasa en función de la radiación fotosintéticamente activa interceptada, que el cultivo acumula a lo largo del ciclo, y de la eficiencia de conversión de la energía lumínica en biomasa. En papa se han elaborado numerosos modelos de crecimiento, desarrollo y rendimiento que aplican este principio (Ingram y McCloud, 1984; Haverkort y Harris, 1987; Jefferies y Heilbronn, 1991; Griffin et al., 1993). La relación entre la radiación interceptada y la biomasa acumulada en el cultivo de papa es lineal, tanto con respecto a la biomasa total, como para la materia seca del tubérculo (Allen y Scott, 1980). Jefferies y MacKerron (1989) encontraron que los factores que más influyen sobre el rendimiento de papa son la intercepción de la radiación y el contenido de agua del tubérculo.

La estructura de la canopia desempeña un papel fundamental en los procesos de intercambio de materia y energía entre la planta y la atmósfera, de tal forma que describir su estado y condición constituye un objetivo prioritario y fundamental en estudios sobre crecimiento vegetal. Tanto el índice de área foliar (IAF) como la distribución angular de las hojas son indicadores usados ampliamente para representar la arquitectura vegetativa de la parte aérea, si bien resultan difíciles de medir directamente (Gordon et al., 1994). Para salvar esta dificultad se han desarrollado instrumentos ópticos que determinan indirectamente el IAF a partir de mediciones de la radiación fotosintéticamente activa (PAR) por encima y por debajo de la canopia (Jonckheere et al., 2004). La utilización de tales herramientas para determinar el IAF en papa no tiene antecedentes en el área del cinturón verde de Córdoba y, en virtud de su capacidad para evaluar el estado de la canopia en forma rápida y no destructiva, configura una opción muy atractiva de poner en práctica.
Reconociendo el papel fundamental del IAF en diversos procesos del crecimiento vegetal, Gordon et al. (1997) evaluaron un modelo que estima la evolución del IAF basado en los principios de intercepción lumínica y la eficiencia de conversión en biomasa, y le incorporaron la influencia del estrés hídrico y las condiciones térmicas para variedades específicas. El IAF, de esta manera, cumple la función de retroalimentación entre las plantas y el régimen de radiación, que de esta forma actúa controlando la capacidad fotosintética del cultivo (Kadaja y Tooming, 2004).

Una manera alternativa de examinar los cambios que experimenta la parte aérea del cultivo durante su ciclo es a partir de mediciones de la fracción de suelo cubierto (Burstall y Harris, 1983; Korva, 1996; Korva y Forbes, 1997). Por otra parte, Burstall y Harris (1983) y Millard et al. (1990) sostienen que la estimación de la cobertura es más fácil de realizar y asumen la existencia de una relación $1: 1$ entre el porcentaje de suelo cubierto $(\% \mathrm{C})$ y la fracción de la radiación fotosintéticamente activa interceptada (fAPAR). Aprovechando la evolución que ha tenido la tecnología digital, es posible realizar actualmente el seguimiento del desarrollo de la canopia por medio de fotografías digitales y determinar el porcentaje de cobertura del suelo aplicando técnicas de interpretación de imágenes (Rodríguez et al., 2000).

Conocer el estado y condición del desarrollo de la canopia constituye una información fundamental en los procedimientos destinados a estimar la productividad de los cultivos, ya sea a partir del relevamiento de campo tradicional, como así también para efectuar evaluaciones por medio de teledetección satelital (Millard et al., 1990). Asimismo, esta información es relevante a los efectos de desarrollar y verificar las rutinas de crecimiento vegetal que sostienen los modelos de simulación de cultivos.

Los objetivos de este ensayo fueron: i) evaluar el empleo y capacidad de un instrumento óptico para determinar el IAF en un cultivo de papa; ii) verificar la utilización del IAF y de la cobertura del suelo como mediciones alternativas del desarrollo de la parte aérea del cultivo, a fin de estimar la intercepción lumínica. 


\section{MATERIALES Y MÉTODOS}

El trabajo se realizó sobre un cultivo de papa (Solanum tuberosum L.) cv. Spunta, durante la campaña (temporada) semitardía del año 2005 (del 4 de febrero al 1 de junio), en un lote (potrero) de producción comercial del cinturón verde de la ciudad de Córdoba ( $31^{\circ} 30^{\prime}$ lat. Sur, $64^{\circ} 08^{\prime}$ long. Oeste, 402 m.s.n.m.). El suelo del sector se clasificó como Haplustol típico, serie Manfredi, limosa fina, mixta, térmica (INTA, 1987), sin limitaciones para la agricultura bajo riego, y aparte de la restricción climática, su condición físico-química no muestra otros impedimentos que limiten el crecimiento de las plantas (Lanfranconi et al., 1987). Una caracterización de la condición edáfica del predio se puede obtener en de la Casa et al. (2003).

El ensayo se llevó a cabo a los efectos de analizar en diferentes marcos de plantación, la evolución de los indicadores del desarrollo de la parte área del cultivo de papa. Después de la emergencia ( $80 \%$ al 28 de febrero), se seleccionaron cuatro camellones que en $20 \mathrm{~m}$ presentaron una densidad de plantación de $6,0 \pm 0,6 \mathrm{pl} \mathrm{m}^{-2}$. De acuerdo al uso generalizado, esta densidad es considerada comercial o normal (tratamiento de densidad comercial (TDC)). Los cuatro surcos inmediatamente contiguos hacia el norte en el mismo sector presentaron una población menor de plantas, raleo que se completó a mano para dejar 3,9 $\pm 0,2 \mathrm{pl} \mathrm{m}^{-2}$ (tratamiento de densidad baja (TDB)). También se hicieron observaciones en un tratamiento de densidad alta (TDA), con una población de 7,9 $\pm 0,7 \mathrm{pl} \mathrm{m}^{-2}$, que se estableció a partir de una segunda pasada de la máquina plantadora el día 10 de febrero. Esta operación tuvo inicialmente la intención de aumentar la densidad de siembra por encima de la comercial, pero, al haberla efectuado desfasada en el tiempo, alteró el proceso normal de brotación. Esta información se utilizó de manera complementaria para verificar las relaciones obtenidas.

Las mediciones de cobertura (\%C) e índice de área foliar (IAF, $\mathrm{m}^{2} \mathrm{~m}^{-2}$ ) se realizaron sobre los camellones centrales, en tanto la extracción de plantas para las determinaciones del área foliar a partir de fotografías, se efectuó sobre los laterales.

El IAF durante el ciclo se midió en forma directa e indirecta. Las mediciones directas se realizaron por un método fotográfico (Rodríguez et al., 2000), determinando la superficie foliar total de las plantas extraídas de un $\mathrm{m}^{2}$ de suelo. La medición indirecta se realizó por medio de un interceptómetro óptico (PAR/LAI Ceptometer, Decagon Devices, Pullman, Washington, USA), efectuando cada fecha entre cinco y siete repeticiones por tratamiento, con la barra dispuesta de manera perpendicular al surco, en distintos sectores de la parcela experimental. Los valores de IAF se calcularon a partir de las lecturas de la radiación fotosintéticamente activa (PAR), medida por encima $\left(\right.$ PAR $\left._{\mathrm{a}}\right)$ y por debajo $\left(\mathrm{PAR}_{\mathrm{d}}\right)$ del dosel, con la siguiente expresión:

$$
\mathrm{IAF}=\frac{\left[\left(1-\frac{1}{2 K}\right) f b-1\right] \operatorname{Ln}\left(\frac{\operatorname{PAR}_{d}}{\operatorname{PAR}_{a}}\right)}{A(1-0,47 f b)}
$$

donde $K$ es el coeficiente de extinción lumínico, que al considerar un parámetro de distribución angular esférico $(\mathrm{x}=1)$, simplifica su cálculo a $K=$ $1 /(2 * \cos v)$, siendo $v$ el ángulo cenital; $f b$ es la fracción de la radiación directa con respecto a la radiación solar recibida, y $A$ es un coeficiente de absorción general del dosel que resulta igual a 0,86 para un valor de absortividad de 0,9 (Campbell, 1986).

También se utilizó el interceptómetro para medir el IAF in situ, esto es en el lugar donde se extrajo la muestra para determinar su valor por fotografías. Las observaciones con el interceptómetro se realizaron sólo bajo condiciones de cielo despejado y durante las horas próximas al mediodía solar. De esta forma el ángulo cenital fue el menor posible, y el factor $f b$ correspondió siempre a fracciones elevadas de radiación solar directa, lo que permitió homogeneizar los datos.

La determinación de la fracción de suelo cubierto por papa $(\% \mathrm{C})$ se realizó también utilizando fotografías digitales y técnicas de interpretación de imágenes. Al igual que para determinar el IAF, se empleó el método de máxima verosimilitud como algoritmo de clasificación de las imágenes (Eastman, 1997). En todas las fechas, el muestreo para obtener la fracción cubierta de suelo consistió de tres fotografías verticales en distintas ubicaciones, tomando el surco entre los camellones centrales como eje longitudinal medio. 


\section{RESULTADOS Y DISCUSIÓN}

En el área del cinturón verde de Córdoba, y en particular con respecto al cultivo de papa, existe poca experiencia en la utilización de instrumentos que midan el IAF a través de la intercepción de la radiación. Por esta razón, se procedió a cotejar el funcionamiento de uno de estos aparatos con respecto a la determinación que se obtiene por medio de fotografías digitales, a modo de método de referencia. La ventaja principal de utilizar un procedimiento fotogramétrico es que realiza la cuantificación directa de la superficie foliar, aunque se trata de un método destructivo y laborioso. La relación entre los valores de IAF que se obtuvieron con los métodos fotogramétrico y del interceptómetro se presenta en la Figura 1.

Como la utilización del interceptómetro constituye una alternativa operativamente sencilla, pero realiza estrictamente una lectura de carácter puntual, se lo empleó de dos maneras. En un caso el IAF del interceptómetro corresponde a un valor medio de cinco lecturas en distintos lugares cercanos al lugar de extracción de la muestra foliar para hacer la determinación fotogramétrica de cada tratamiento, mientras que el otro caso resulta de un valor medio sólo del lugar donde se la extrajo.

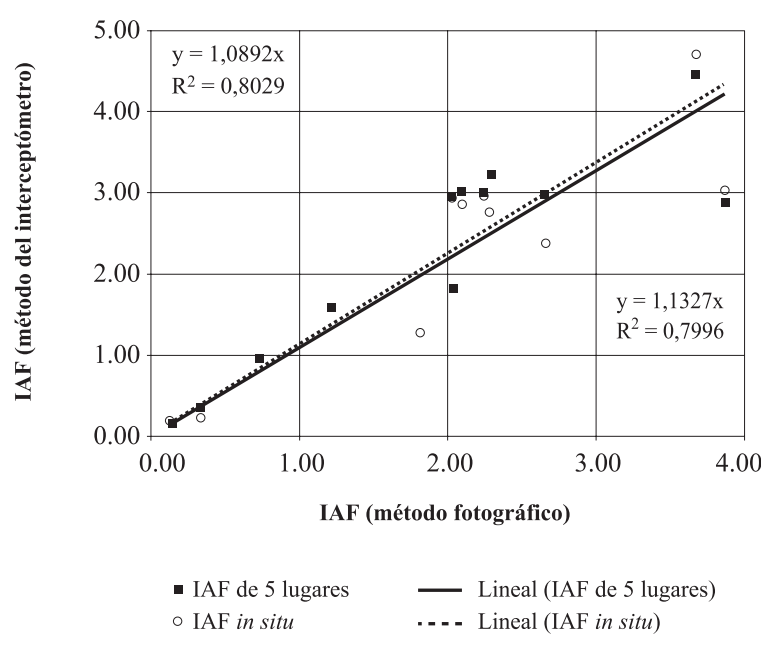

Figura 1. Relación entre el índice de área foliar (IAF) $\left(\mathrm{m}^{2} \mathrm{~m}^{-2}\right)$ obtenido por medio de fotografías digitales y con el instrumental óptico.

Figure 1. Relationship between leaf area index (IAF) $\left(\mathrm{m}^{2} \mathrm{~m}^{-2}\right)$ obtained by means of digital pictures and optic instrument.
La representación muestra el carácter lineal de la relación en ambos casos, con el coeficiente angular de la recta de regresión cercano a la unidad. El coeficiente de determinación bajo las dos modalidades aplicadas ronda un valor de 0,80 $(\mathrm{P}<0,01)$. Esta dispersión en exceso se puede explicar porque la determinación destructiva del IAF a los efectos de la medición fotográfica procede de un único sector $\left(1 \mathrm{~m}^{2}\right)$, en contraposición a mediciones más repetidas que se obtienen con el instrumento óptico. También se advierte que la dispersión tiende a aumentar a medida que el tamaño de la muestra se hace más grande, lo que puede ser consecuencia de la pérdida de precisión del interceptómetro en condiciones de saturación lumínica. En la Figura 2 se observa que para valores de IAF mayores a 3, el cambio que muestra la intercepción de la radiación es muy reducido. Por otra parte, no se aprecian diferencias de consideración entre las dos formas de medir el IAF con el interceptómetro.

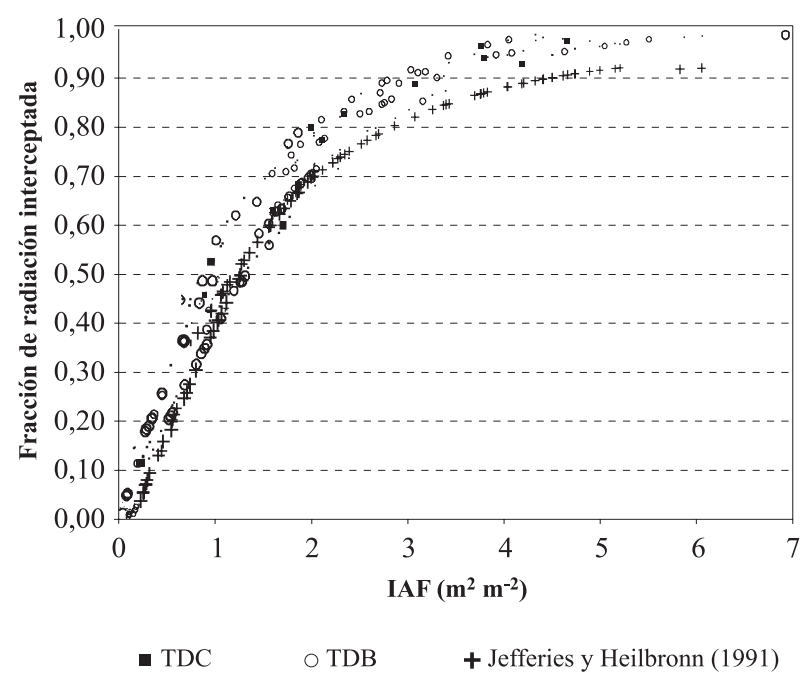

Figura 2. Relación entre el índice de área foliar (IAF) calculado y la fracción de radiación interceptada $\left(1-P_{A} R_{a} / P_{A} R_{d}\right)$ en los tratamientos de densidad comercial (TDC) y baja (TDB) para diferentes momentos del ciclo. Se han agregado también los valores de la fracción que estima el modelo de Jefferies y Heilbronn (1991).

Figure 2. Relationship between the calculated leaf area index (IAF) and the fraction of intercepted radiation (1-PAR $/ \mathbf{P A R}_{\mathrm{d}}$ ) in commercial (TDC) and low (TDB) density treatments at different moments of the cycle. The fraction values that were estimated according to Jefferies and Heilbronn's (1991) model have also been added. 
La determinación del IAF que realiza el interceptómetro se basa en la inversión de un modelo simplificado de Norman y Campbell (1989), que asume una relación exponencial negativa entre la radiación solar interceptada y el IAF. A fin de mostrar el comportamiento de esta relación a partir de las mediciones de radiación fotosintéticamente activa por encima y por debajo de la canopia, se presenta la Figura 2. En todo el transcurso del ensayo se observa que la relación entre la radiación interceptada $\left(1-\mathrm{PAR}_{\mathrm{a}} / \mathrm{PAR}_{\mathrm{d}}\right.$ ) y el IAF calculado se mantuvo uniforme, tanto en el sentido de comparar las dos densidades de plantación, como así también durante los diferentes momentos del ciclo, es decir con valores de área foliar bajos y altos. La uniformidad de la curva se justifica por cuanto los valores de IAF fueron calculados en condiciones muy semejantes durante el ciclo, tanto con respecto al ángulo cenital como a la fracción fb. Por su parte, el coeficiente de extinción lumínica (k) se asumió invariable durante el ciclo para una distribución foliar esférica. Este aspecto debería ser revisado de manera particular, por cuanto en papa fue sugerida su variación sistemática durante el ciclo respecto al desarrollo del área foliar (Gordon et al., 1997), al igual que bajo la influencia de la densidad de siembra o la distancia entre filas de plantas, como también se ha observado en distintos cultivos anuales (Rosenthal et al., 1993; Flénet et al., 1996).

La Figura 2 también muestra que cuando los valores de IAF son superiores a 3, la fracción de radiación interceptada cambia muy poco, de forma tal que su contribución para captar energía luminosa y aprovecharla para producir materia seca resulta, a partir de este valor, progresivamente menos importante. Estas curvas no difieren mayormente de la propuesta por el modelo de Jefferies y Heilbronn (1991), en particular cuando el IAF es inferior a 3 y asumiendo el carácter genérico que presentan las estimaciones del modelo.

El desarrollo de la parte aérea del cultivo de papa durante la temporada 2005 tuvo la evolución temporal que muestra la Figura 3, para los tratamientos de densidad de plantación comercial y baja. La dinámica que mostró la cobertura fue representada hasta que el cultivo alcanza a cerrar el surco, lo que aproximadamente corresponde al final de la etapa de crecimiento expolineal (Goudriaan y van Laar, 1995). Por su parte, la variación del IAF ex- presa de manera equivalente el crecimiento de la parte aérea hasta alcanzar cobertura completa, y luego comienza a declinar gradualmente durante la etapa de senescencia.

Estas curvas exhiben un comportamiento típico que, para las de cobertura, es semejante al presentado por Millard et al. (1990), aunque en este caso alcanzan la condición de cobertura completa un poco antes, alrededor del día 50 después de la emergencia, probablemente porque el ciclo transcurre desde verano hacia otoño, con niveles térmicos elevados al comienzo del ciclo. La condición de cobertura del tratamiento de densidad comercial (TDC) durante todas las fechas observadas fue siempre superior al de baja densidad (TDB), pero estas diferencias fueron disminuyendo a medida que el cultivo se aproximó a cobertura completa.

Por su parte, el desarrollo del IAF tuvo un comportamiento similar al que presentan Allen y Scott (1980) para diferentes densidades de siembra. La densidad superior alcanza más rápido en la temporada un IAF de 3 , como así también tiene un pico máximo más elevado de 4,5, pero que rápidamente desaparece. En el caso de la densidad más

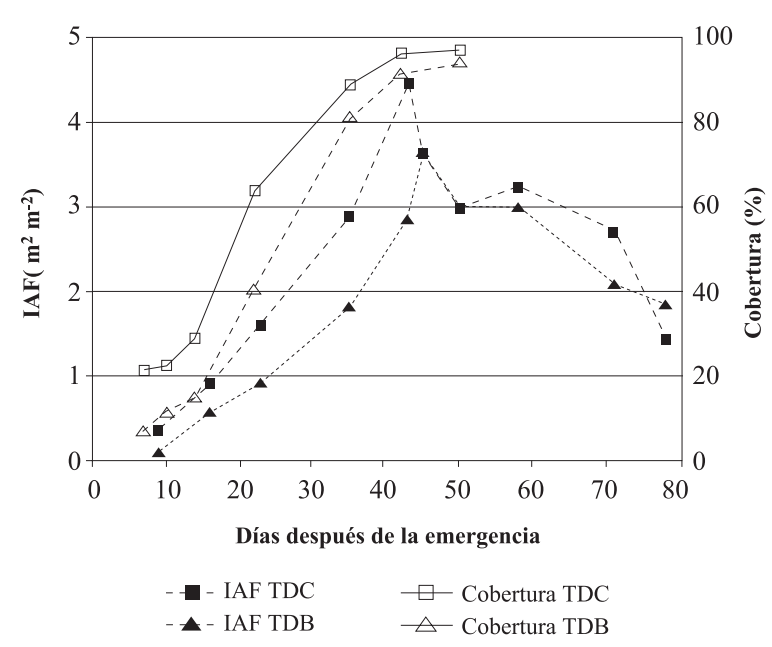

Figura 3. Variación que experimentaron el porcentaje de suelo cubierto $(\% \mathrm{C})$ y el índice de área foliar (IAF) en el transcurso del ciclo de papa para los tratamientos de densidad de siembra comercial (TDC) y baja (TDB).

Figure 3. Ground cover $(\% \mathrm{C})$ and leaf area index (IAF) variation, experienced during the potato cycle for commercial (TDC) and low (TDB) density treatments. 
baja el máximo resulta inferior $(3,5)$ y ocurre ligeramente más tarde. En ambos casos son picos de corta duración, asociado al comportamiento de vuelco del cultivo (Allen y Scott, 1980), de forma tal que tienen presumiblemente poca importancia desde el punto de vista de la intercepción de la radiación.

Las curvas de IAF presentan un crecimiento de tres fases, similar al propuesto por Gordon et al. (1997) en su modelo de desarrollo de la canopia para variedades específicas de papa. Asimismo, una vez alcanzado el valor de IAF máximo, la tendencia es que posteriormente no existan diferencias importantes entre el IAF de ambas densidades de siembra, de manera similar a lo reportado por Allen y Scott (1980) en la etapa final del ciclo.

Una verificación a la propuesta de Burstall y Harris (1983), que consideran posible estimar la intercepción de la luz utilizando tanto mediciones de cobertura del suelo como de IAF, fue realizada analizando la relación entre ambas expresiones. Los resultados se presentan en la Figura 4.

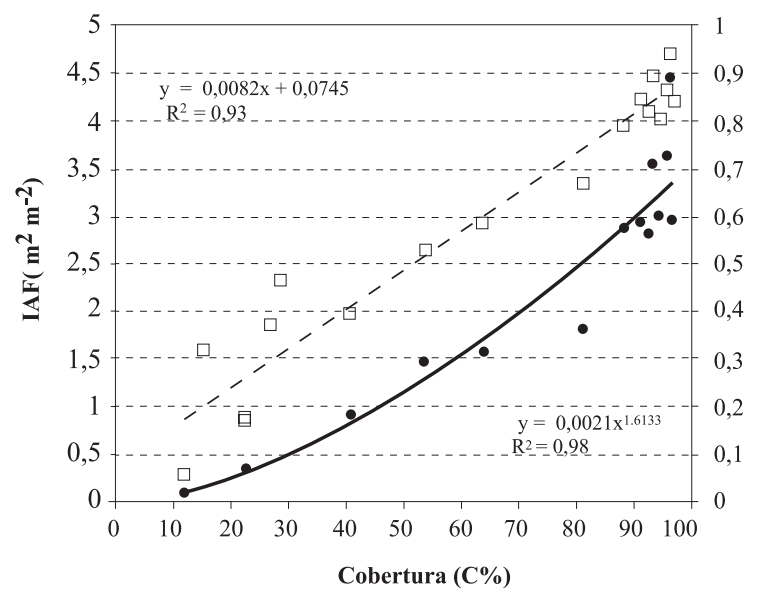

- IAF calculado Interceptómetro

- fAPAR

- - . Lineal (fAPAR)

— Potencial (IAF calculado Interceptómetro)

Figura 4. Relación entre el porcentaje de cobertura (\%C), el índice de área foliar (IAF) medido con el instrumento óptico y la fracción de la radiación interceptada (fAPAR).

Figure 4. Relationship between ground cover $(\% \mathrm{C})$, the leaf area index (IAF) measured with the optic instruments and the intercepted radiation fraction (fAPAR).
Hasta que el cultivo alcanza cobertura máxima, que no llega a $100 \%$ en ninguno de los tratamientos (Jefferies y Heilbronn, 1991), la representación gráfica muestra la existencia de una relación lineal, con un coeficiente de ajuste $\left(\mathrm{R}^{2}=0,93\right)$ altamente significativo $(\mathrm{P}<0,01)$, entre el porcentaje de cobertura y la fAPAR. Por su parte, la relación exponencial entre $\% \mathrm{C}$ y el IAF revela que en cobertura completa el cv. Spunta presenta valores de IAF próximos a $3(\mathrm{P}<0,01)$. Las curvas de ajuste de los dos tratamientos por separado no presentan diferencias respecto al comportamiento del conjunto, por lo que se lo puede considerar característico. La relación entre $\% \mathrm{C}$ y fAPAR no resulta exactamente $1: 1 \mathrm{y}$, si bien la utilización directa del valor de cobertura es más sencilla, de esta forma se producirían ligeras subestimaciones sobre la radiación interceptada al comienzo del ciclo y sobreestimaciones algo más importantes hacia el final, como se muestra en la Figura 5. Elaborada con información independiente (obtenida del tratamiento TDA), se aprecia en este caso que la curva de ajuste entre \%C y fAPAR presenta, respecto de la curva identidad, valores más elevados al principio del ciclo y luego más bajos al aproximarse a cobertura completa.

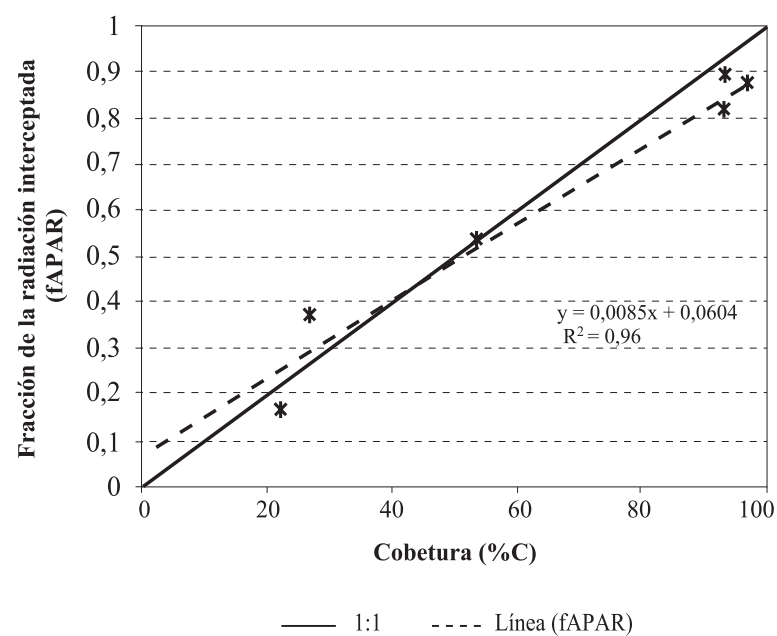

Figura 5. Relación entre la cobertura del suelo (\%C) y la fracción de radiación interceptada (fAPAR) para una muestra independiente (TDA) respecto a la curva de identidad.

Figure 5. Relationship between ground cover (\%C) and the intercepted radiation fraction (fAPAR) for an independent sample (TDA) and the 1:1 line. 


\section{CONCLUSIONES}

El instrumento óptico utilizado ofrece buenas perspectivas para obtener mediciones confiables del IAF en papa, pero su uso adecuado requiere de una alta intensidad de muestreo (no menos de cinco repeticiones), la condición despejada del cielo (valores de $\mathrm{fb}>0,84$ ), el ángulo cenital reducido (mediciones realizadas próximas al mediodía solar) y valo- res de IAF inferiores a 3. La relación entre el porcentaje de suelo cubierto y la radiación interceptada no resulta exactamente $1: 1 \mathrm{y}$, si bien la utilización directa del valor de cobertura es más sencilla, de esta forma se producirían ligeras subestimaciones sobre la radiación interceptada al comienzo del ciclo y sobreestimaciones algo más importantes hacia el final.

\section{LITERATURA CITADA}

Allen, E.J., and R.K. Scott. 1980. An analysis of growth of the potato crop. J. Agri. Sci. (Cambridge) 94:583606.

Burstall, L., and P.M. Harris. 1983. The estimation of percentage light interception from leaf area index and percentage ground cover in potatoes. J. Agri. Sci. (Cambridge) 100:241-244.

Campbell, G.S. 1986. Extinction coefficients for radiation in plant canopies calculated using an ellipsoidal inclination angle distribution. Agric. For. Meteorol. 36:317-321.

De la Casa, A.C., G.G. Ovando, A.R Rodríguez, L. Bressanini, y E. Buffa. 2003. Evaluación espacial del nitrógeno disponible del suelo en un cultivo de papa cv. Spunta en Córdoba, Argentina. Agric. Téc. (Chile) 63:311-318.

Eastman, R.J. 1997. Clasification of remotely sensed imagery. p. 1-29. In IDRISI: User's guide Idrisi for Windows. Clark University, Worcester, Massachusetts, USA.

Flénet, F., J.R. Kiniry, J.E. Board, M.E. Westgate, and D.C. Reicosky. 1996. Row spacing effects on light extinction coefficients of corn, sorghum, soybean, and sunflower. Agron. J. 88:185-190.

Gordon, R., D.M. Brown, and M.A. Dixon. 1994. Nondestructive estimation of potato leaf area index using a fish-eye radiometer. Potato Res. 37:393-402.

Gordon, R., D.M. Brown, and M.A. Dixon. 1997. Estimating potato leaf area index for specific cultivars. Potato Res. 40:251-266.

Goudriaan, J., and H.H. van Laar. 1995. Modeling potential crop growth processes. 238 p. Kluwer Academic Publishers, Dordrecht, The Netherlands.

Griffin, T.S., B.S. Johnson, and J.T. Ritchie. 1993. A simulation model for potato growth and development: Substor-potato Version 2.0. International Benchmark Sites Network for Agrotechnology Transfer. IBSNAT Research Report Series 02.29 p. University of Hawaii, College of Tropical Agriculture an Human Resources, Honolulu, Hawaii, USA.
Haverkort, A.J., and P.M. Harris. 1987. A model for potato growth and yield under tropical highland conditions. Agric. For. Meteorol. 39:271-282.

Ingram, K.T., and D.E. McCloud. 1984. Simulation of potato growth and development. Crop Sci. 24:21-27

INTA. 1987. Plan mapa de suelos. Hoja 3163-32, Oncativo. Carta de suelos de la República Argentina. 82 p. INTA y SMAGyRR, Córdoba, Argentina.

Jefferies, R.A., and T.D. Heilbronn. 1991. Water stress as a constraint on growth in the potato crop. 1. Model development. Agric. For. Meteorol. 53:185-196.

Jefferies, R.A., and D.K.L. Mac Kerron. 1989. Radiation interception and growth of irrigated and droughted potato (Solanum tuberosum L.). Field Crop Res. 22:101-112.

Jonckheere, I., S. Fleck, K. Nackaerts, B. Muys, P. Coppin, M. Weiss, and F. Baret. 2004. Review of methods for in situ leaf area index determination. Part I. Theories, sensors and hemispherical photography. Agric. For. Meteorol. 121:19-35.

Kadaja, J., and H. Tooming. 2004. Potato production model based on principle of maximum plant productivity. Agric. For. Meteorol. 127:17-33.

Korva, J.T. 1996. Grids in ground cover measurements. Potato Res. 39:533-540.

Korva, J.T., and G.A. Forbes. 1997. A simple and lowcost method for leaf area measurement of detached leaves. Exp. Agric. 33:65-72.

Lanfranconi, L.E., E.A. Tuda, M. Buteler, W. Robledo, M. Fontán, y R. Beretta. 1987. Situación de contexto del área central bajo riego de la provincia de Córdoba. Volumen I: Tomo I y II. Instituto Nacional de Tecnología Agropecuaria (INTA), Estación Experimental Agropecuaria Manfredi, Córdoba, Argentina.

Millard, P., G.G. Wright, M.J. Adams, R.V. Birnie, and P. Withworth. 1990. Estimation of light interception and biomass of the potato (Solanum tuberosum L.) from reflection in the red and near-infrared spectral bands. Agric. For. Meteorol. 53:19-31. 
Monteith, J.L. 1977. Climate and the efficiency of crop production in Britain. Philos. Trans. R. Soc. London, Ser. B. 281:277-294.

Monteith, J.L. 1994. Validity of the correlation between intercepted radiation and biomass. Agric. For. Meteorol. 68:213-220.

Norman, J.M., and G.S. Campbell. 1989. Canopy structure. Plant physiological ecology: Field methods and instrumentation. p. 301-325. In R.E. Pearcy, J.R. Ehleringer, H.A. Mooney, and P.W. Rundel (eds.). Chapman and Hall, London, United Kingdom.
Rodríguez, A., A. de la Casa, R. Accietto, L. Bressanini, y G. Ovando. 2000. Determinación del área foliar en papa (Solanum tuberosum L., var. Spunta) por medio de fotografías digitales conociendo la relación entre el número de pixeles y la altura de adquisición. Rev. Bras. Agrometeorol. 8:215-221.

Rosenthal, W.D., T.J. Gerik, and L.J. Wade. 1993. Radiation-use efficiency among grain sorghum cultivars and plant densities. Agron. J. 85:703-705. 